\title{
Variants of meningiomas: a review of imaging findings and clinical features
}

\author{
Akira Kunimatsu ${ }^{1}$ Natsuko Kunimatsu ${ }^{2} \cdot$ Kouhei Kamiya $^{3} \cdot$ Masaki Katsura $^{3}$ • \\ Harushi Mori ${ }^{1} \cdot$ Kuni Ohtomo $^{1,4}$
}

Received: 30 March 2016 / Accepted: 14 April 2016 / Published online: 2 May 2016

(C) Japan Radiological Society 2016

\begin{abstract}
Meningiomas are common neoplasms that frequently occur in the brain and spine. Among the 15 histological subtypes of meningiomas in the WHO classification, the incidence of meningothelial meningiomas is the highest, followed by fibrous and transitional meningiomas. These three subtypes account for approximately $80 \%$ of all meningiomas, and thus could be regarded as typical meningiomas. For this reason, other uncommon histological subtypes may be considered as imaging variants, and diagnosis is often challenging for radiologists solely based on imaging features of typical meningiomas. In addition to the histological subtypes, meningiomas arising in atypical locations could be easily mistaken for other lesions more commonly observed in those locations. The purpose of this article is to review characteristic clinical and imaging findings of uncommon meningiomas, including histological variants and meningiomas occurring in relatively rare locations.
\end{abstract}

Keywords Meningioma $\cdot$ Classification $\cdot$ Magnetic resonance imaging $\cdot \mathrm{X}$-ray computed tomography

Akira Kunimatsu

akrk-tky@umin.ac.jp

1 Department of Radiology, Graduate School of Medicine, The University of Tokyo, 7-3-1, Hongo, Bunkyo-ku, Tokyo 113-8655, Japan

2 Department of Radiology, International University of Health and Welfare, Mita Hospital, Tokyo, Japan

3 Department of Radiology, The University of Tokyo Hospital, Tokyo, Japan

4 International University of Health and Welfare, Otawara, Japan

\section{Introduction}

Meningiomas are meningothelial cell neoplasms, which typically attach to the inner surface of the dura matter [1]. They account for $27 \%$ of all primary brain tumors in Japan, and middle-aged women are most commonly affected [2]. According to the WHO classification of central nervous system (CNS) tumors published in 2007 [1], most meningiomas are benign and categorized as WHO grade I. However, certain histological subtypes are associated with less favorable clinical outcomes and categorized as WHO grade II or III, indicating malignancy. Meningothelial meningioma is the most common subtype (Fig. 1), followed by fibrous (Fig. 2) and transitional subtypes (Fig. 3). These three subtypes account for approximately $80 \%$ of all meningiomas (Table 1) [3], which radiologists would identify as typical meningiomas. In contrast, each of the other histological subtypes constitutes only $1-3 \%$ of all meningiomas, respectively.

Meningiomas usually form sessile or lentiform, wellcircumscribed, extra-axial mass lesions with broad-based dural attachment. They characteristically demonstrate hyperdensity on non-contrast $\mathrm{CT}$, iso- to hypointensity on T1-weighted, and iso- to hyperintensity on T2-weighted images [4]. Meningothelial, fibrous, and transitional meningiomas can show variable intensities on diffusionweighted images; thus, apparent diffusion coefficient (ADC) values may vary greatly. Most of them will demonstrate no diffusion restriction or facilitation on diffusionweighted images, compared with the brain parenchyma [5]. Uniform and strong enhancement is typically seen after contrast administration. Dural tails [6], hyperostosis in the underlying bones, linear internal flow voids, and calcification are also often observed in meningioma 
Fig. 1 Meningothelial meningioma in a 29-year-old woman. a Axial T2-weighted image shows a well-circumscribed, extra-axial mass on the left cerebral convexity. The mass shows homogeneous hyperintensity compared with the gray matter. b, c Axial diffusionweighted image and the corresponding apparent diffusion coefficient map show no diffusion restriction or facilitation on the mass. d Axial post-contrast T1-weighted image shows the homogeneously enhancing mass with the dural tail sign (arrow). e Axial bone window CT image shows thickening of the underlying bones (arrows). $\mathbf{f}$ Left lateral oblique projection of the left external carotid angiogram depicts the characteristic spoke-wheel pattern of the mass (arrow)
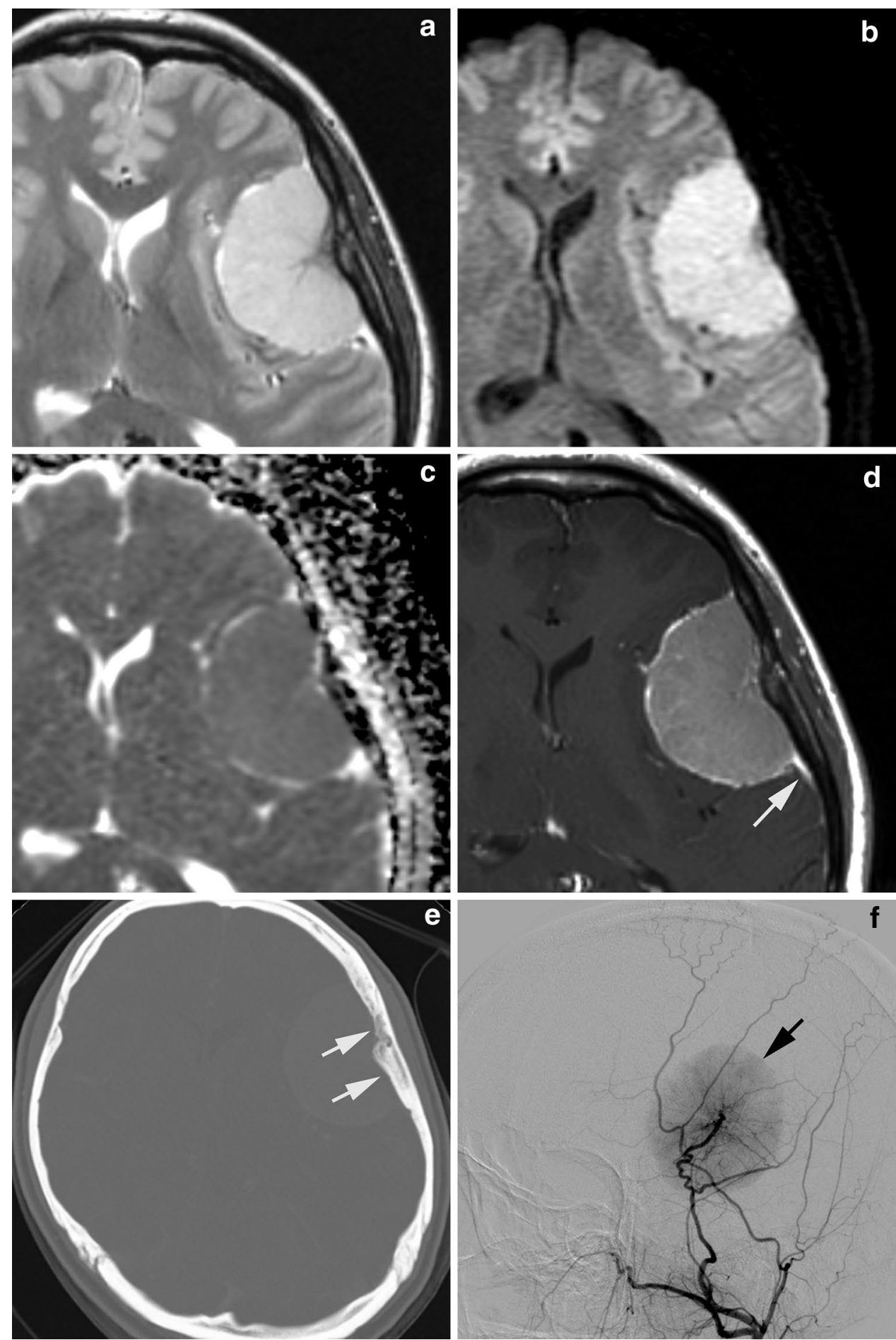

lesions. Peritumoral brain edema can occur when the tumor becomes large. On trans-catheter angiograms, meningiomas will show characteristic sunburst or spokewheel appearances (Fig. 1). These typical imaging findings are likely to reflect common histological subtypes of meningiomas. Therefore, some of these imaging features may be applicable to uncommon histological variants of meningiomas and some may not. The wide variety of imaging findings may cause confusion in the diagnosis of meningiomas.

Meningiomas with WHO grade II or III are associated with high recurrence rates and mortality. Successful gross total resection significantly decreases the rate of recurrence, compared with subtotal resection [7]. In WHO grade I meningiomas, tumor consistency, associated with tumor histology, is an important factor in determining the extent 


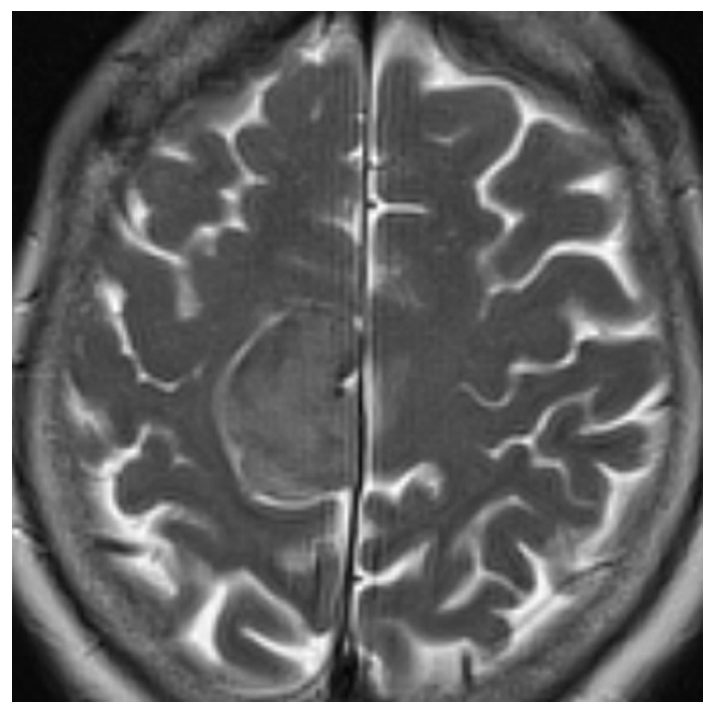

Fig. 2 Fibrous meningioma in a 69-year-old woman. Axial T2-weighted image shows a relatively hypointense mass on the falx cerebri, likely reflecting the abundant collagen accumulated on histology

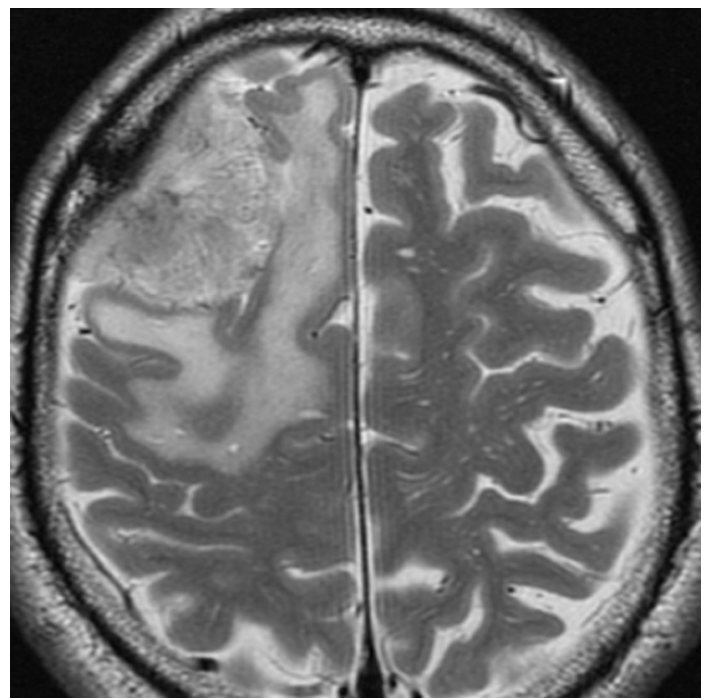

Fig. 3 Transitional meningioma in a 71-year-old man. Axial T2-weighted image shows a mass with mixed iso- and hyperintensity on the right cerebral convexity, possibly representing the typical coexistence of meningothelial and fibrous patterns on histology

of tumor resection and surgical outcome. Therefore, preoperative imaging information regarding histologic grade and subtype of meningioma is crucial [8].

In this article, we review the characteristics of clinical and imaging findings of uncommon meningioma variants (as are summarized in Table 2). We hope that this article will enhance and organize the knowledge of meningiomas for radiologists.
Table 1 Incidence of histological subtypes of meningioma

\begin{tabular}{lcll}
\hline Subtype & Incidence (\%) & Subtype & Incidence (\%) \\
\hline WHO grade I & & WHO grade II \\
Meningothelial & 57.8 & Chordoid & 1.4 \\
Fibrous & 11.1 & Clear cell & 1.1 \\
Transitional & 10.4 & Atypical & 3.4 \\
Psammomatous & 3.8 & & \\
Angiomatous & 1.6 & WHO grade III \\
Microcystic & 0.3 & Papillary & 1.1 \\
Secretory & 1.5 & Rhabdoid & 2.3 \\
Lymphoplasmacyte-rich & 1.1 & Anaplastic & 1.4 \\
Metaplastic & 1.6 & & \\
\hline
\end{tabular}

The incidence of each histological subtype was calculated based on the reported findings in reference No. 3

\section{Uncommon histological subtypes of meningiomas}

\section{Psammomatous meningioma}

Psammomatous meningioma is characterized by the predominance of psammoma bodies, when compared with typical meningothelial meningiomas [1]. This histological variant corresponds to WHO grade I. Psammomatous meningiomas share common locations with typical meningiomas in the cranium [3], but in the literature, many case reports described intraspinal psammomatous meningiomas, especially in the thoracic spine [9]. CT reveals calcification diffusely or at the periphery of the tumor. T1-weighted images typically demonstrate iso-intensity with or without low signals. On T2-weighted images, the tumor commonly shows low intensity, but may show iso- to high intensity as well. Such hypointensities on MR images do not always correspond to calcifications seen on CT, although calcifications are expected to cause signal reduction [9]. ADC values of psammomatous meningiomas likely fall within the range of those of typical meningiomas [5]. Post-contrast MR images show solid and strong enhancement in many cases (Fig. 4). Fewer cases can have weak contrast enhancement at the periphery of the tumor.

\section{Angiomatous meningioma}

Angiomatous meningioma is a rare histological subtype characterized by the predominance of blood vessels, corresponding to WHO grade I [1]. A slight male predominance may be a clinical characteristic, in contrast to the female predominance of typical meningiomas. On non-contrast CT, the tumor shows slightly high attenuation. The tumor is typically hypointense on T1-weighted images, hyperintense on T2-weighted images, and 
Table 2 Summary of imaging features of uncommon meningiomas by histological subtypes

\begin{tabular}{|c|c|c|c|c|}
\hline Histological subtype & $\mathrm{T} 2 \mathrm{WI}$ & DWI & CE-T1WI & Other notable features \\
\hline \multicolumn{5}{|l|}{ WHO grade I } \\
\hline Psammomatous & Hypo > iso, hyper & Similar to brain & Homo $>$ peripheral & $\begin{array}{l}\text { Dense calcification on } \mathrm{CT} \text {, predilec- } \\
\text { tion for thoracic spine }\end{array}$ \\
\hline Angiomatous & Hyper, with edema & Possibly facilitated & Homo & Prominent hypervascularity \\
\hline Microcystic & Hyper, with edema & Possibly facilitated & Homo $>$ reticular & Obviously hypo on T1WI \\
\hline Secretory & Hyper, with edema & Possibly facilitated & Homo & Predilection for skull base \\
\hline $\begin{array}{l}\text { Lymphoplasmacyte- } \\
\text { rich }\end{array}$ & Hyper to iso, with edema & Possibly restricted & Indistinct margin & En plaque meningioma \\
\hline Metaplastic & \multicolumn{4}{|c|}{ Various, depending on mesenchymal components } \\
\hline \multicolumn{5}{|l|}{ Grade II } \\
\hline Chordoid & Hyper & Facilitated diffusion & Homo $>$ hetero & $\begin{array}{l}\text { Occasionally resembles Castleman's } \\
\text { disease }\end{array}$ \\
\hline Clear cell & $\begin{array}{l}\text { Mixed iso- to hyper, with cyst and } \\
\text { edema }\end{array}$ & Similar to brain & Hetero & $\begin{array}{l}\text { Relatively young patients, predilec- } \\
\text { tion for CP angle }\end{array}$ \\
\hline Atypical & Mixed hyper to hypo & Restricted diffusion & Hetero & Increased permeability \\
\hline \multicolumn{5}{|l|}{ Grade III } \\
\hline Papillary & Iso $>$ hyper, with cyst and edema & Restricted diffusion & Hetero & Lobulated margin \\
\hline Rhabdoid & Hyper $>$ iso, with cyst & No published data & Homo $>$ hetero & Pediatric patients \\
\hline Anaplastic & Mixed hyper to hypo & Restricted diffusion & Hetero & Increased permeability \\
\hline
\end{tabular}

T1WI T1-weighted imaging, T2WI T2-weighted imaging, DWI diffusion-weighted imaging, CE-T1WI contrast-enhanced T1-weighted imaging, hypo hypointense, iso isointense, hyper hyperintense, homo homogeneous enhancement, hetero heterogeneous enhancement, $C P$ angle cerebellopontine angle, and "> " indicates "more often observed than"

Fig. 4 Psammomatous meningioma in a 73-year-old woman. a Sagittal T2-weighted image shows a well-circumscribed, intra-dural, and extra-axial mass at the Th10 vertebra level. The mass shows a homogeneous hypointensity compared with the spinal cord. b Sagittal postcontrast T1-weighted image demonstrates homogeneous enhancement. c Sagittal reformatted contrast-enhanced CT image reveals dense calcification throughout the mass
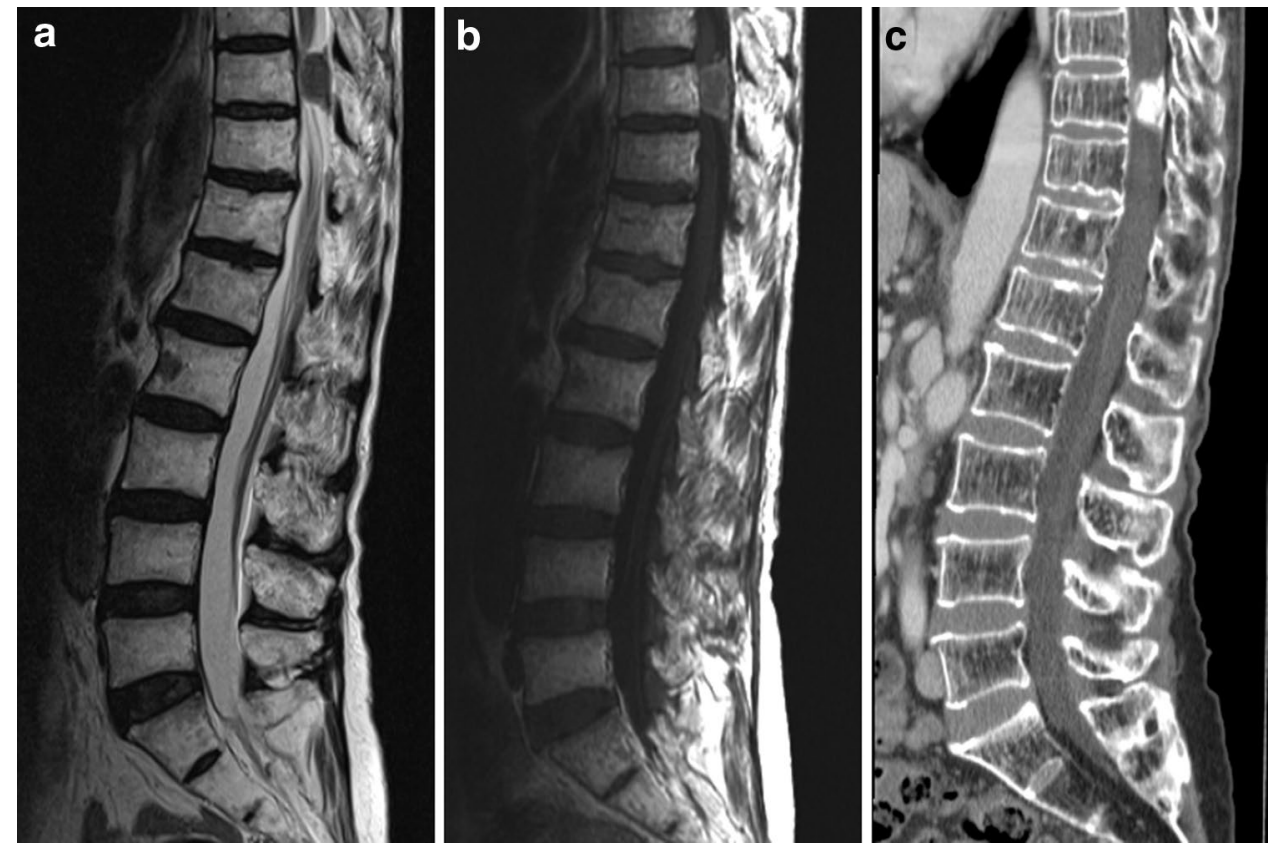

slightly hypointense on diffusion-weighted images [10]. Post-contrast MR images mostly demonstrate vivid enhancement because of the prominent hypervascularity. Homogeneous enhancement is seen in $85 \%$ of the tumor, whereas $15 \%$ of the tumor can show heterogeneous enhancement or cyst formation. Internal flow voids can be detected in almost all cases and feeding arteries are easily depicted on MR angiography. Peritumoral brain edema is usually prominent even for a small tumor (Fig. 5). 

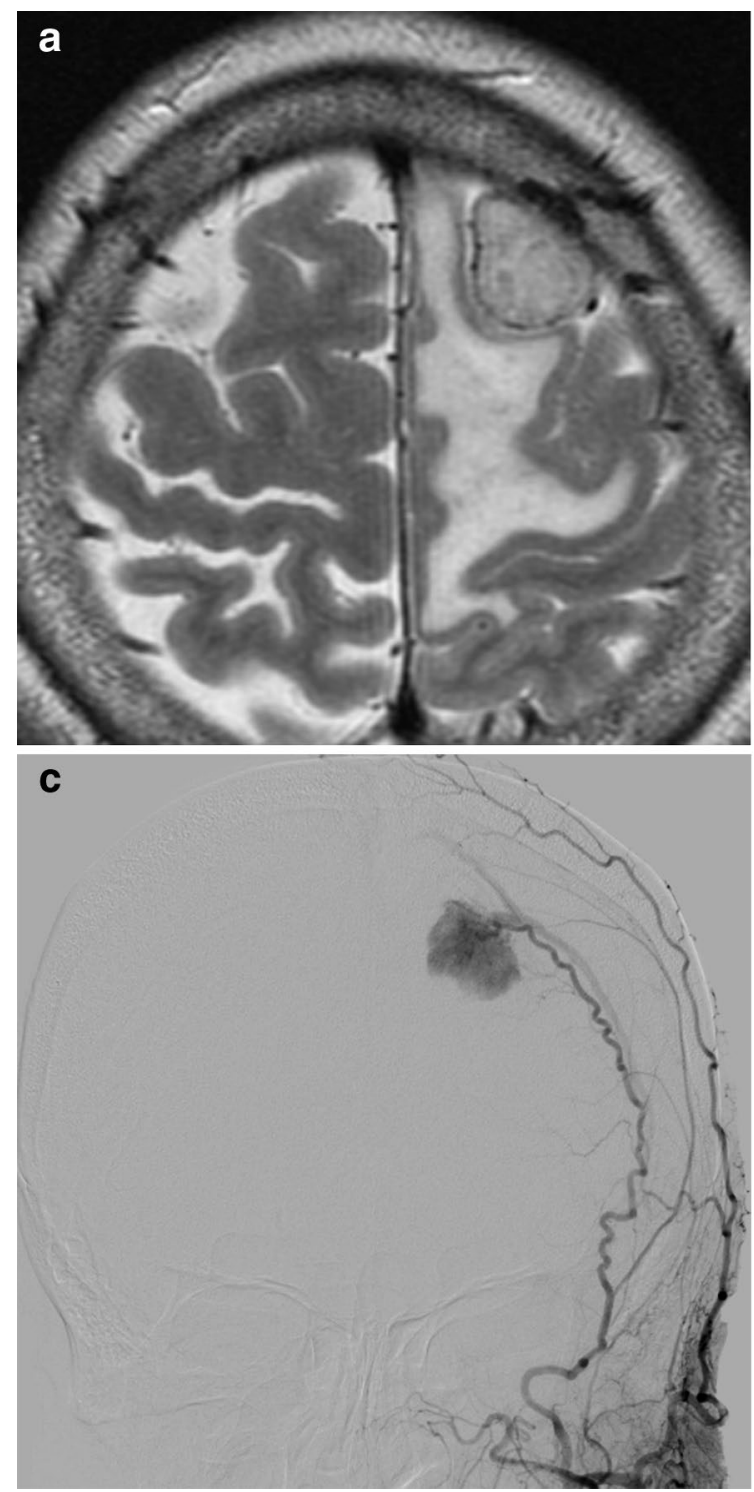

Fig. 5 Angiomatous meningioma in a 65-year-old woman. a Axial T2-weighted image shows a well-circumscribed, extra-axial mass on the left cerebral convexity. The mass shows a homogeneous hyperintensity, and peritumoral brain edema is prominent even with the small

\section{Microcystic meningioma}

Microcystic meningioma is the rarest histological subtype, characterized by the formation of intercellular microcystic spaces [1], and falls within the category of WHO grade I. Microcystic meningiomas show low $(50 \%)$ to iso-attenuation $(50 \%)$ on non-contrast CT. Microcystic meningiomas characteristically demonstrate distinct low intensity on T1-weighted images and high intensity on T2-weighted images [11]. Chen and colleagues reported that obvious hypointensity on T1-weighted images was the only significant predictor of microcystic meningioma on multivariate analysis [12]. ADC values of microcystic meningiomas

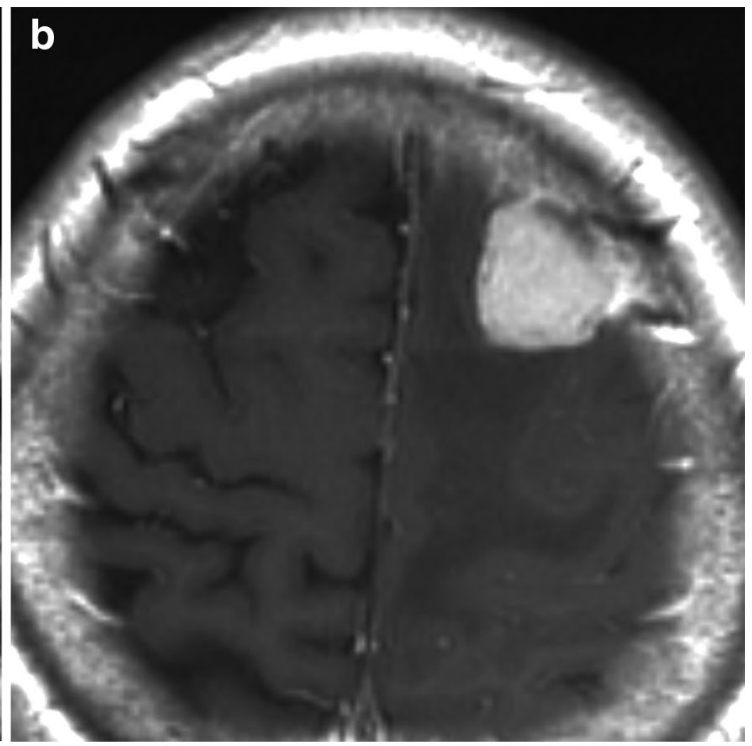

size of the mass. b Axial post-contrast T1-weighted image demonstrates strong and homogeneous enhancement. c Postero-anterior projection of the left external carotid angiogram shows the hypervascular mass with early venous return

may be relatively high, compared with those of typical meningiomas [5]. Reticular enhancement may be characteristic on post-contrast MR images and can be seen in $30 \%$ of the tumor, but diffuse, strong enhancement is more common (Fig. 6). Peritumoral brain edema can be demonstrated in up to $88 \%$ of the tumor, and the edema is usually marked.

\section{Secretory meningioma}

Secretory meningioma is characterized by an intracellular lumen containing an eosinophilic secretion which is called a pseudo-psammoma body, corresponding to WHO grade 

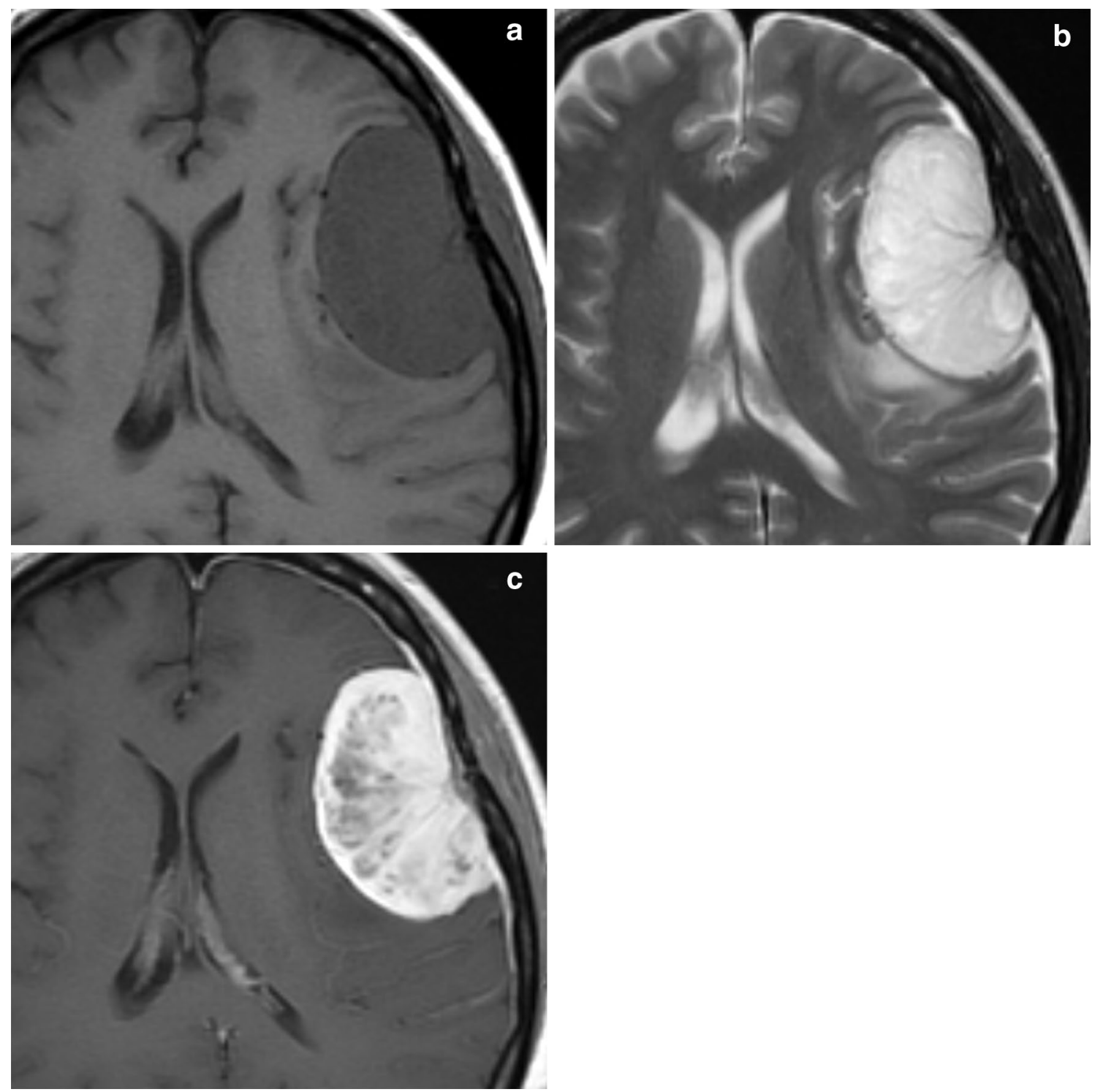

Fig. 6 Microcystic meningioma in a 46-year-old woman. a Axial pre-contrast T1-weighted image shows a well-circumscribed, extraaxial mass on the left cerebral convexity. The mass characteristically shows hypointensity compared with the gray matter. b Axial

I [1]. It shows a predilection for the skull base and forms a mass with smooth, distinct margins in $83 \%$ of cases but rarely with irregular margins. On T1-weighted images, the tumor shows non-specific iso- to low intensity, but on T2-weighted images, it characteristically shows high intensity in up to $96 \%$ of the cases [13]. Secretory meningiomas may exhibit relatively high ADC values, compared with typical meningiomas [5]. Post-contrast images show homogeneous and marked enhancement of the tumor. Peritumoral brain edema can be seen in $60 \%$ of cases (Fig. 7). Cyst formation is rare.
T2-weighted image demonstrates the hyperintense mass with internal linear strands. c Axial post-contrast T1-weighted image shows strong and reticular enhancement

\section{Lymphoplasmacyte-rich meningioma}

This rare variant contains extensive chronic inflammatory infiltrates and corresponds to WHO grade I [1]. Clinical features include a predilection for relatively young patients, often with secondary hypergammaglobulinemia and anemia [14]. The lymphoplasmacyte-rich subtype characteristically arises as an en-plaque meningioma on the dura, with irregular forms and unclear tumor boundaries. Invasion to the adjacent brain tissue can occur. Typically, the tumor is hyperdense on non-contrast CT and iso- to hypointense 
on T1-weighted images. T2-weighted images show mixed hyper- to iso-intensity more often than iso- to hypointensity. Diffusion restriction can be seen [15]. Post-contrast MR images show strong and homogeneous enhancement with indistinct margins (Fig. 8), likely reflecting tumor extension and inflammatory cell infiltration [16]. Cyst formation can be detected in $30 \%$ and peritumoral brain edema in nearly $100 \%$ of the cases.

\section{Clear cell meningioma}

Clear cell meningioma corresponds to WHO grade II and is characteristically composed of polygonal cells with clear cytoplasm and rich interstitial collagen [1]. Clinical characteristics include a predilection for relatively young patients and the cerebellopontine angle as the location of the tumor. Recurrence and metastasis are common. Non-contrast CT shows variable density but most often high density of the tumor. The tumor demonstrates iso- to slightly low intensity on T1-weighted and inhomogeneous, mixed iso- to high intensity on T2-weighted images. ADC values of clear cell meningiomas may fall within the range of those of typical meningiomas [15]. The tumor shows strong and heterogeneous contrast enhancement more often than homogeneous enhancement (Fig. 9) [17]. Cysts are seen in $60 \%$ and peritumoral brain edema in $78 \%$ of the cases. Osteolysis is seen more often than hyperostosis of the underlying bones.

\section{Atypical and anaplastic meningiomas}

Anaplastic meningioma has a clearly malignant cytology that resembles carcinoma or high-grade sarcoma, thus corresponding to WHO grade III. Atypical meningioma demonstrates intermediate malignant features between grade I meningioma and anaplastic meningioma and corresponds to WHO grade II [1]. Clinically, these subtypes more commonly affect relatively old patients, with a male predominance, in contrast to typical benign meningiomas. General imaging features include indistinct tumor-brain interfaces, irregular tumor margins, inhomogeneous tumor
Fig. 7 Secretory meningioma in a 73-year-old woman. a Coronal T2-weighted image shows a round, extra-axial mass on the left middle cranial fossa (arrows). Peritumoral brain edema is prominent even with the small size of the mass. b Coronal post-contrast T1-weighted image with fat suppression demonstrates strong and homogeneous enhancement. Secretory meningioma commonly arises at the skull base
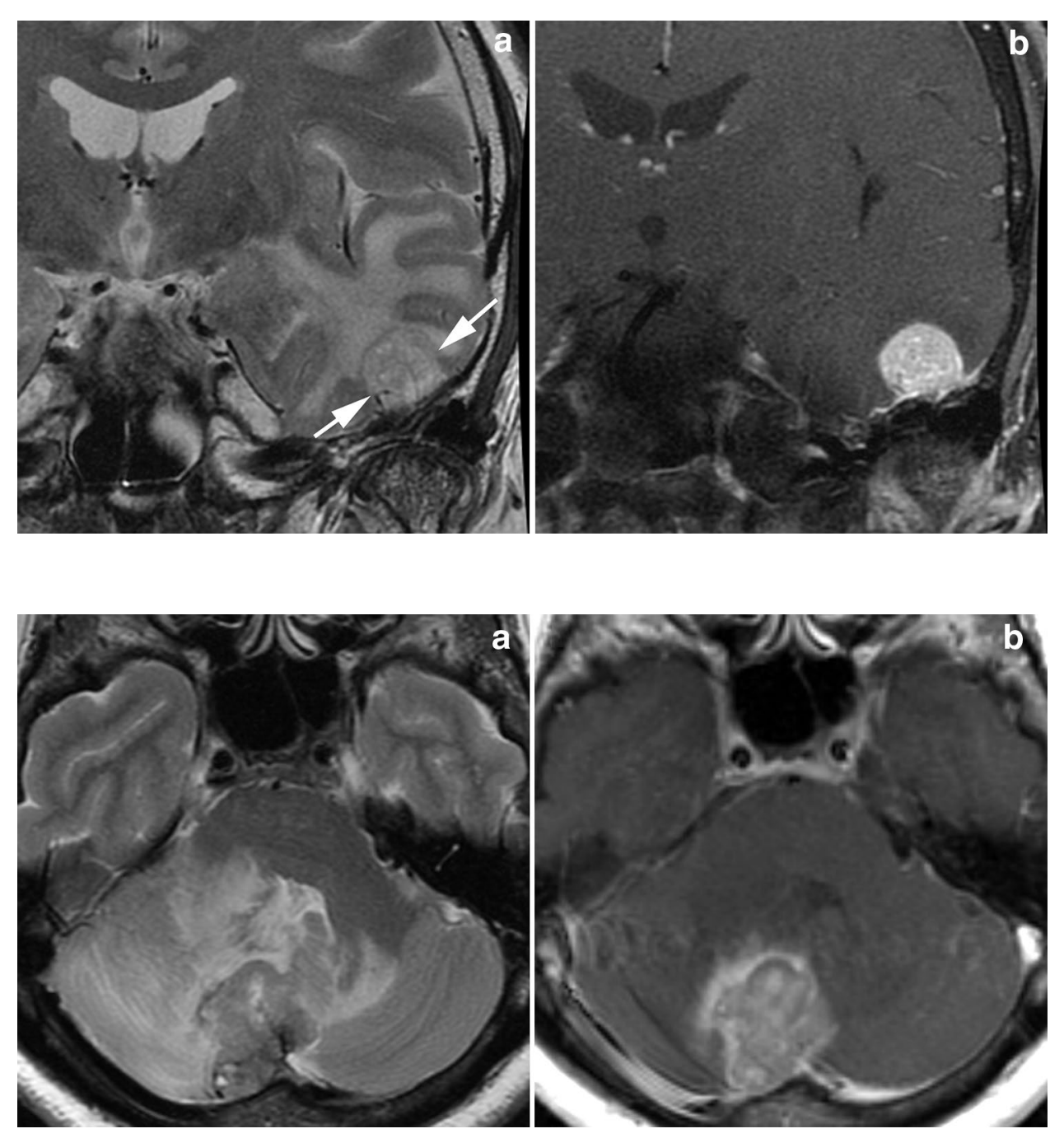

Fig. 8 Lymphoplasmacyte-rich meningioma in a 56-year-old woman. a Axial T2-weighted image shows an irregularshaped mass, attaching the cerebellar convexity to the falx cerebelli. Peritumoral brain edema is marked. b Axial postcontrast T1-weighted image demonstrates heterogeneous enhancement with unclear tumor boundaries, likely reflecting tumor extension and inflammatory cell infiltrates along the pial surface of the cerebellum 
Fig. 9 Clear cell meningioma in a 47-year-old woman. a Axial T2-weighted image shows an irregular-shaped mass (arrows) in front of the medulla oblongata. The right vertebral artery is deviated posteriorly and the epicenter of the mass is located in the extra-axial space. The mass shows an inhomogeneous appearance with mixed iso- and hyperintensity. b Axial postcontrast T1-weighted image demonstrates heterogeneous enhancement
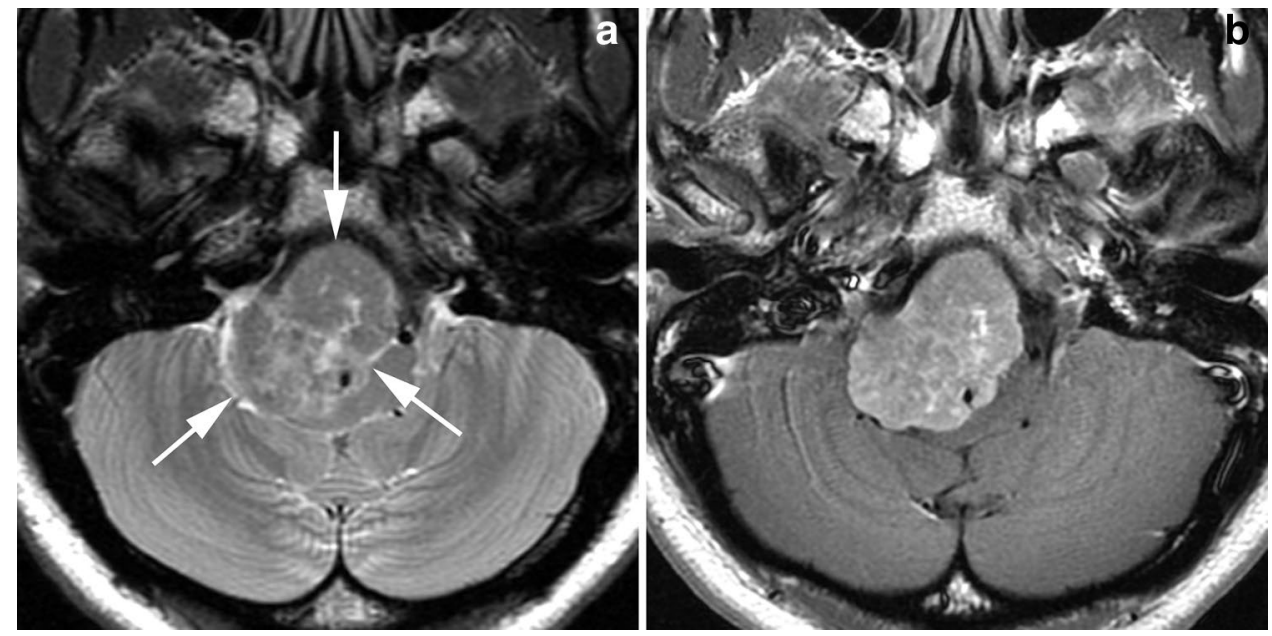

appearances, and the lack of capsule-like enhancement at the tumor margins [18]. Diffusion-weighted images most likely show restricted diffusion [5, 19, 20]. On dynamic susceptibility contrast perfusion MR images, the relative cerebral blood volume increases in the area of peritumoral brain edema with a delay in contrast arrival [21]. The volume transfer contrast, $K^{\text {trans }}$, obtained with dynamic contrast-enhanced perfusion MR imaging, usually shows higher values in atypical meningiomas compared with those in typical meningiomas, which is a useful indicator in their differentiation [22]. Other common features of atypical and anaplastic meningiomas include necrosis, calcification, prominent peritumoral brain edema, and bone destruction (Fig. 10).

\section{Other histological variants}

Other histological variants include metaplastic meningioma (WHO grade I), chordoid meningioma (WHO grade II), and papillary and rhabdoid meningiomas (WHO grade III) [1]. Metaplastic meningioma shows striking focal or diffuse mesenchymal components. The tumor may contain osseous, cartilaginous, myxoid, lipomatous, or xanthomatous tissue. Metaplastic meningiomas are often named based on the dominant mesenchymal components, such as lipomatous or xanthomatous meningioma [23]. Controversies still remain over whether the tumor is truly a metaplastic form of meningioma [24]. Imaging characteristics are variable, depending on its mesenchymal components. Chordoid meningioma characteristically has tissue histologically similar to chordomas. The tumor can present with Castleman's disease when it occurs in childhood [25]. A recent study showed that facilitated diffusion, shown as elevations of $\mathrm{ADC}$ values, is highly characteristic of chordoid meningioma [15]. Papillary meningioma is characterized by a perivascular pseudo-papillary pattern on histology.
Fig. 10 Anaplastic meningioma in a 72-year-old woman. a Coronal reformatted post-contrast CT image shows an osteolytic mass of the left middle cranial fossa to the left infratemporal fossa (arrows). The center of the mass demonstrates weaker enhancement compared with the periphery, possibly representing necrosis. b Axial T2-weighted image shows the inhomogeneous high intensity of the mass infiltrating the surrounding soft tissue. c, $\mathbf{d}$ Axial diffusion-weighted image and the corresponding apparent diffusion coefficient map show diffusion restriction in the medial part of the lesion, possibly reflecting the high cellularity of the mass. e Axial post-contrast T1-weighted image shows heterogeneous enhancement

Common imaging features include irregular margins, heterogeneous enhancement, moderate or marked irregular peritumoral edema, and cyst formation [26]. Rhabdoid meningioma features the predominance of sheets of rhabdoid cells. Generally, the prognosis is poor, and many case reports are available on pediatric patients in the literature. On CT and MRI, the tumor demonstrates severe peritumoral edema in $75 \%$, cyst formation in $38 \%$, and mixed bone destruction and hyperostosis in $33 \%$ of the cases [27].

\section{Variations in locations of meningiomas}

Meningiomas arise from meningothelial cells or arachnoid cap cells, and the tumors more frequently occur where the cells are abundant. The arachnoid granulations or villi have large numbers of arachnoid cap cells, and therefore are common sites for the origin of meningiomas. The villi are concentrated along the dural venous sinuses, and this feature of the anatomy likely explains the reason why meningiomas commonly occur adjacent to the sinuses. Common locations of intracranial or juxtacranial meningiomas include: convexity (20-34\%); parasagittal and falcine (18-22\%); sphenoid and middle cranial fossa 

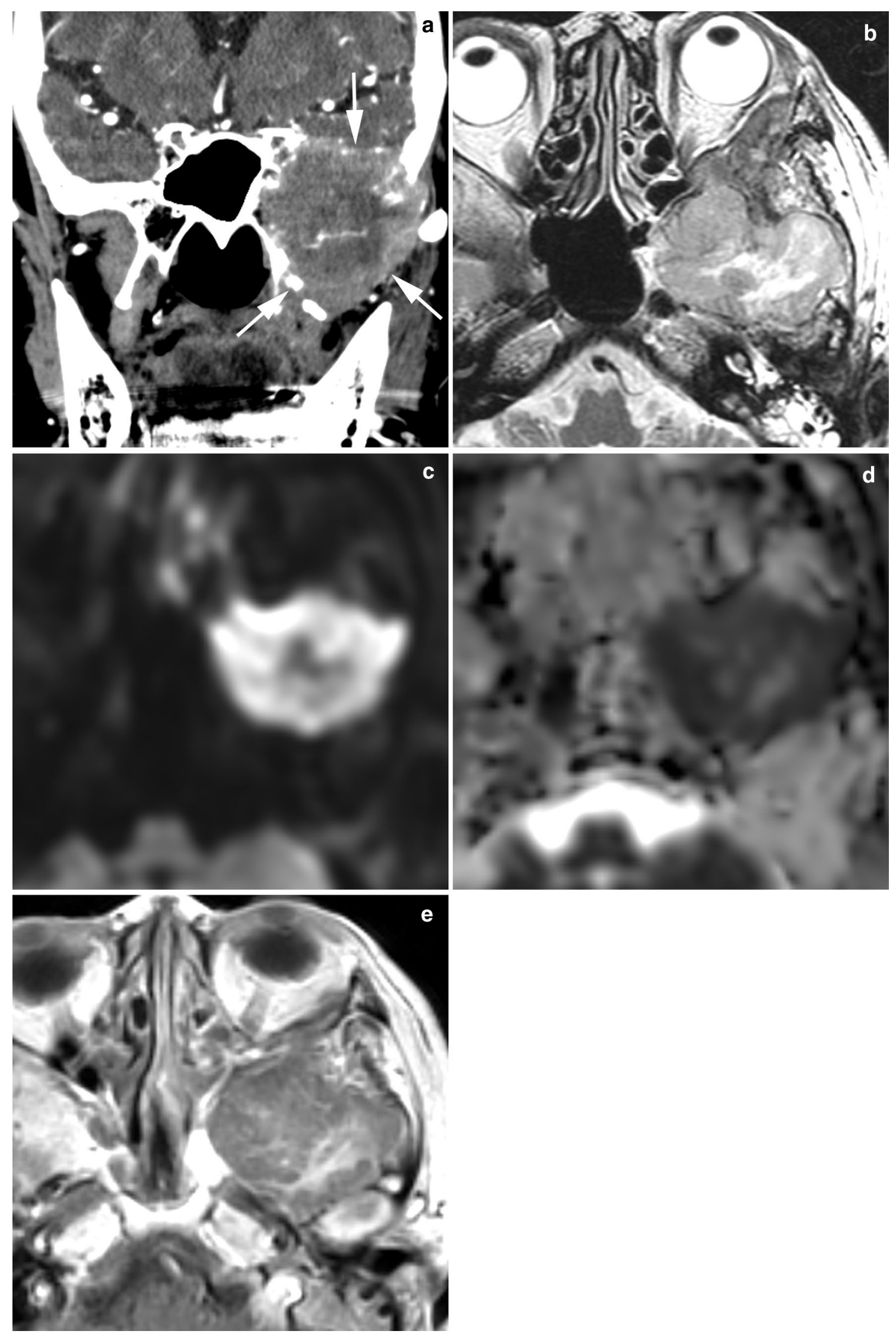


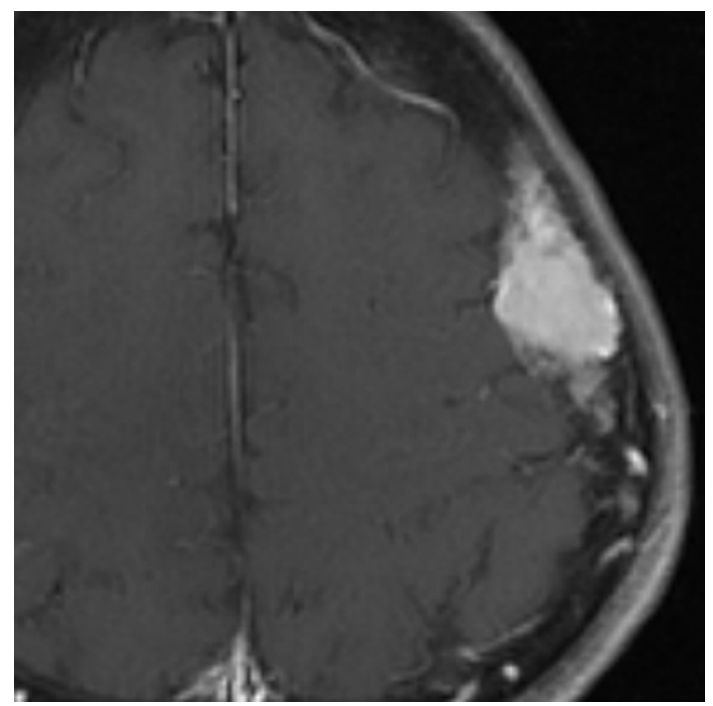

Fig. 11 Primary intraosseous (intradiploic) meningioma in a 68-year-old woman. Axial fat-suppressed post-contrast T1-weighted image shows a homogeneously enhanced mass in the left parietal bone

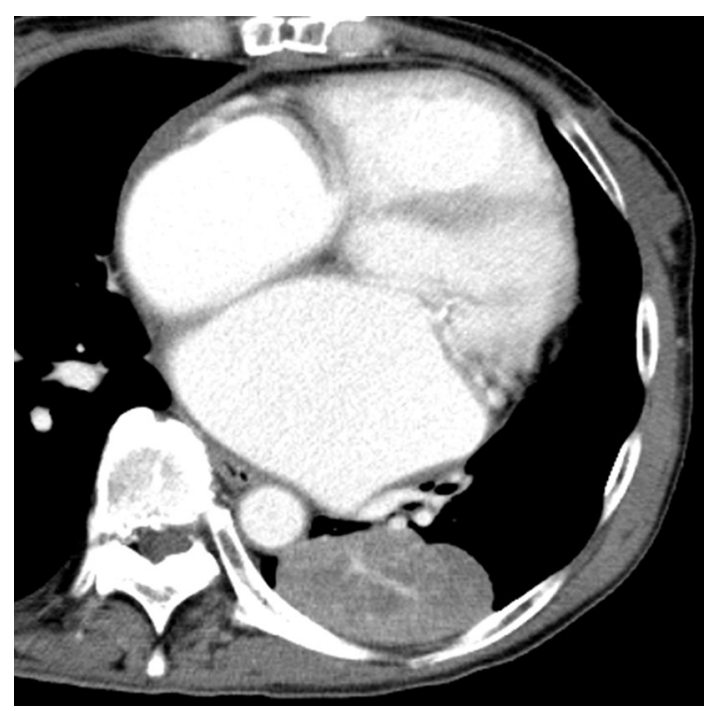

Fig. 12 Metastatic pulmonary meningioma in a 59-year-old woman. Axial post-contrast CT image shows a homogeneously enhanced mass in the left hemi-thorax

(17-25\%); frontobasal (10\%); posterior fossa (9-15\%) including the tentorium cerebelli (2-4\%), cerebellar convexity $(5 \%)$, cerebellopontine angle $(2-4 \%)$, intraventricular $(2-5 \%)$, and orbital $(<1-2 \%)$ [4]. The incidence of intraspinal meningiomas is about $1 / 9$ of that of intracranial meningiomas.

Meningiomas developing in ectopic locations account for only $1-2 \%$ of all meningiomas at most. Ectopic meningiomas can be either primary or metastatic. Although primary ectopic meningiomas are rare, most of them arise in the skull and the head and neck regions. Other much rarer locations include the lung, mediastinum, retroperitoneum, pelvis, and extremities [28]. Primary ectopic meningiomas of the skull and the head and neck regions are considered to arise from the arachnoid cells around the cranial nerves exiting the skull or from the arachnoid cells disseminated during the formation of the skull. Possible explanations of the origin of meningiomas at sites distant from the neuraxis include congenital, ectopic, meningothelial cell rests and meningothelial differentiation from pluripotent mesenchymal cells. Imaging findings can be variable. Intradiploic meningiomas can show both osteoblastic and osteolytic lesions (Fig. 11) [29]; thus, possible differential diagnoses on CT and MRI include metastasis, multiple myeloma, osteosarcoma, fibrous dysplasia, and intraosseous hemangioma. Moreover, as for the head and neck region, because of their rarity, it may be impractical to suggest ectopic meningiomas as the working diagnoses before histological confirmation. Differential diagnoses should be modified depending on the tumor locations and imaging findings, but could generally include hypervascular tumors.

Extraneural metastases from meningiomas are extremely rare, accounting for approximately $0.1 \%$ of all meningiomas. The lung is the most frequent site of metastases (61\%) (Fig. 12), followed by the liver, lymph node, bone, pleura, and mediastinum [30]. A case with multiple metastatic lesions is uncommon. Many cases present with one or two metastatic lesions, with or without the concurrent local recurrence of meningioma [31]. Dural sinus invasion, a history of craniotomy, specific histological subtype (papillary meningioma), and higher WHO grade could be risk factors for metastasis, but none of them has been established [30]. No imaging findings have been established as specific for metastatic meningiomas, and many extraneural metastases are found incidentally on radiological examination.

\section{Conclusion}

The diagnosis of meningioma is often challenging when encountering imaging variants. However, many rare histological variants have their own imaging or clinical features, compared with typical meningiomas. We believe that knowledge of these features will help radiologists make a diagnosis of meningioma with more confidence.

\section{Compliance with ethical standards}

Conflict of interest The authors declare that they have no conflict of interest. 
Ethical statement This review work was approved by the institutional review board at the University of Tokyo and informed consent was waived.

\section{References}

1. WHO classification of tumours of the central nervous system. 4th ed. In: Bosman FT, Jaffe ES, Lakhani SR, Ohgaki H, editors. Lyon: International agency for research on cancer (IARC); 2007.

2. Report of brain tumor registry of Japan (1984-2000). Neurol Med Chir (Tokyo). 2009;49 Suppl:PS1-96.

3. Bhat AR, Wani MA, Kirmani AR, Ramzan AU. Histologicalsubtypes and anatomical location correlated in meningeal brain tumors (meningiomas). J Neurosci Rural Pract. 2014;5(3):244-9.

4. Buetow MP, Buetow PC, Smirniotopoulos JG. Typical, atypical, and misleading features in meningioma. Radiographics. 1991;11(6):1087-106.

5. Santelli L, Ramondo G, Della Puppa A, Ermani M, Scienza R, d'Avella D, et al. Diffusion-weighted imaging does not predict histological grading in meningiomas. Acta Neurochir (Wien). 2010;152(8):1315-9 (discussion 9).

6. Tokumaru A, O'Uchi T, Eguchi T, Kawamoto S, Kokubo T, Suzuki M, et al. Prominent meningeal enhancement adjacent to meningioma on Gd-DTPA-enhanced MR images: histopathologic correlation. Radiology. 1990;175(2):431-3.

7. Ko KW, Nam DH, Kong DS, Lee JI, Park K, Kim JH. Relationship between malignant subtypes of meningioma and clinical outcome. J Clin Neurosci Off J Neurosurg Soc Australas. 2007;14(8):747-53.

8. Wang S, Kim S, Zhang Y, Wang L, Lee EB, Syre P, et al. Determination of grade and subtype of meningiomas by using histogram analysis of diffusion-tensor imaging metrics. Radiology. 2012;262(2):584-92.

9. Lee JW, Lee IS, Choi KU, Lee YH, Yi JH, Song JW, et al. CT and MRI findings of calcified spinal meningiomas: correlation with pathological findings. Skelet Radiol. 2010;39(4):345-52.

10. Liu Z, Wang C, Wang H, Wang Y, Li JY, Liu Y. Clinical characteristics and treatment of angiomatous meningiomas: a report of 27 cases. Int J Clin Exp Pathol. 2013;6(4):695-702.

11. Paek SH, Kim SH, Chang KH, Park CK, Kim JE, Kim DG, et al. Microcystic meningiomas: radiological characteristics of 16 cases. Acta Neurochir (Wien). 2005;147(9):965-72 (discussion 72).

12. Chen CJ, Tseng YC, Hsu HL, Jung SM. Microcystic meningioma: importance of obvious hypointensity on T1-weighted magnetic resonance images. J Comput Assist Tomogr. 2008;32(1):130-4.

13. Wang DJ, Xie Q, Gong Y, Wang Y, Cheng HX, Mao Y, et al. Secretory meningiomas: clinical, radiological and pathological findings in 70 consecutive cases at one institution. Int J Clin Exp Pathol. 2013;6(3):358-74.

14. Yongjun L, Xin L, Qiu S, Jun-Lin Z. Imaging findings and clinical features of intracal lymphoplasmacyte-rich meningioma. J Craniofac Surg. 2015;26(2):e132-7.

15. Pond JB, Morgan TG, Hatanpaa KJ, Yetkin ZF, Mickey BE, Mendelsohn DB. Chordoid meningioma: differentiating a rare
World Health Organization grade II tumor from other meningioma histologic subtypes using MRI. AJNR Am J Neuroradiol. 2015;36(7):1253-8.

16. Liu JL, Zhou JL, Ma YH, Dong C. An analysis of the magnetic resonance imaging and pathology of intracal lymphoplasmacyterich meningioma. Eur J Radiol. 2012;81(5):968-73.

17. Wang $X Q$, Huang MZ, Zhang H, Sun FB, Tao BB, Feng BH, et al. Clear cell meningioma: clinical features, CT, and MR imaging findings in 23 patients. J Comput Assist Tomogr. 2014;38(2):200-8.

18. Kawahara Y, Nakada M, Hayashi Y, Kai Y, Hayashi Y, Uchiyama $\mathrm{N}$, et al. Prediction of high-grade meningioma by preoperative MRI assessment. J Neurooncol. 2012;108(1):147-52.

19. Hakyemez B, Yildirim N, Gokalp G, Erdogan C, Parlak M. The contribution of diffusion-weighted MR imaging to distinguishing typical from atypical meningiomas. Neuroradiology. 2006;48(8):513-20.

20. Surov A, Ginat DT, Sanverdi E, Lim CC, Hakyemez B, Yogi $A$, et al. Use of diffusion-weighted imaging in differentiating between malignant and benign meningiomas. A multicenter analysis. World Neurosurg. 2015 Oct 31 (epub ahead of print).

21. Zhang H, Rodiger LA, Shen T, Miao J, Oudkerk M. Perfusion MR imaging for differentiation of benign and malignant meningiomas. Neuroradiology. 2008;50(6):525-30.

22. Yang S, Law M, Zagzag D, Wu HH, Cha S, Golfinos JG, et al. Dynamic contrast-enhanced perfusion MR imaging measurements of endothelial permeability: differentiation between atypical and typical meningiomas. AJNR Am J Neuroradiol. 2003;24(8):1554-9.

23. Ishida M, Fukami T, Nitta N, Iwai M, Yoshida K, Kagotani A, et al. Xanthomatous meningioma: a case report with review of the literature. Int J Clin Exp Pathol. 2013;6(10):2242-6.

24. Colnat-Coulbois S, Kremer S, Weinbreck N, Pinelli C, Auque J. Lipomatous meningioma: report of 2 cases and review of the literature. Surgical Neurol. 2008;69(4):398-402 (discussion).

25. Jeon CJ, Kim MJ, Lee JS, Lee JH, Kong DS, Shin HJ, et al. Castleman's disease associated with a cerebellar chordoid meningioma and intestinal lymphangiectasia. Child's Nerv Syst ChNS Off J Int Soc Pediatr Neurosurg. 2010;26(11):1647-52.

26. Yu XR, Jun Z, Zhang BY, Huang WY, Bo Y, Tan WL, et al. Magnetic resonance imaging findings of intracranial papillary meningioma: a study on eight cases. Clin Imaging. 2014;38(5):611-5.

27. Kim EY, Weon YC, Kim ST, Kim HJ, Byun HS, Lee JI, et al. Rhabdoid meningioma: clinical features and MR imaging findings in 15 patients. AJNR Am J Neuroradiol. 2007;28(8):1462-5.

28. Nur S, Chuang L, Ramaswamy G. Primary extracranial meningioma of the pelvis: a light microscopic, immunohistochemical, and ultrastructural study. Gynecol Oncol. 2006;103(2):745-8.

29. Budhdeo S, Ibrahim RA, Hofer M, Gillies M. Primary intraosseous osteoblastic meningioma. JRSM Short Rep. 2011;2(6):52.

30. Erman T, Hanta I, Haciyakupoglu S, Zorludemir S, Zeren H, Gocer AI. Huge bilateral pulmonary and pleural metastasis from intracranial meningioma: a case report and review of the literature. J Neurooncol. 2005;74(2):179-81.

31. Kaminski JM, Movsas B, King E, Yang C, Kronz JD, Alli PM, et al. Metastatic meningioma to the lung with multiple pleural metastases. Am J Clin Oncol. 2001;24(6):579-82. 